J Ästhet Chir 2019 · 12:51-58 https://doi.org/10.1007/s12631-018-0168-x Online publiziert: 6. Dezember 2018 (c) Der/die Autor(en) 2018 CrossMark

Lymphgefäße sind am anatomischen Präparat nicht oder kaum sichtbar, sodass sich Untersuchungen der Lymphgefäße äußerst schwierig gestalten. Bereits im 19. Jahrhundert wurde damit begonnen, den Verlauf der Lymphgefäße darzustellen, indem an Leichen Quecksilber in Lymphgefäße injiziert wurde. Quecksilber besitzt eine besonders hohe Kapillarität zu Lymphgefäßen und breitet sich daher rasch innerhalb dieser aus. Da heutzutage die Verwendung von Quecksilber aufgrund seiner hohen Toxizität verboten ist und bislang keine Substanz mit vergleichbarer Kapillarität gefunden werden konnte, sind Untersuchungen der Lymphgefäße an anatomischen Präparaten beinahe unmöglich.

Bezüglich der embryonalen Entwicklung des Lymphgefäßsystems herrscht bis heute Uneinigkeit. Es gilt als gesichert, dass das lymphatische System wesentlich später als das Blutgefäßsystem entsteht [4]. Die Abstammung der Lymphgefäße ist jedoch noch nicht gänzlich geklärt. Hierzu existieren zumindest 2 Theorien. Eine Theorie besagt, dass die Lymphgefäße unabhängig von den Blutgefäßen aus Lücken innerhalb des Mesenchyms entstehen. Einer anderen Theorie zufolge sollen die Lymphgefäße aus dem Endothel der Venen aussprossen.

\section{Allgemeines}

Das Lymphsystem arbeitet parallel zum Venensystem als „Drainage- und Transportsystem“ $[1,6]$. Die initialen Lymphgefäße sind die sog. Lymphkapillaren oder Lymphsinus. Diese sind einfache Endothelschläuche und weisen noch keine Klappen auf. Diese Lymphkapillaren

Friedrich Anderhuber ist vor der Veröffentlichung dieses Beitrags verstorben.

\author{
Ulrike Pils|' · Friedrich Anderhuber \\ 'Gottfried Schatz Zentrum, Makroskopische und klinische Anatomie, Medizinische Universität Graz, Graz, \\ Österreich
}

\title{
Anatomie des Lymphsystems
}

(Lymphsinus) erhalten die Lymphe aus dem „prälymphatischen Kanalsystem“" des Interstitiums. Über eine interzelluläre Lücke („schwingender Zipfel“) gelangt die Lymphe aus dem Interstitium in die Lymphkapillaren (• Abb. 1).

Das Lymphgefäßsystem gliedert sich in ein oberflächliches und ein tiefes System. Das oberflächliche System ist innerhalb des subkutanen Gewebes - also oberflächlich zur Faszie - lokalisiert, drainiert die Lymphe der Haut und des subkutanen Gewebes und verläuft großteils parallel zu den subkutanen Venen. Das tiefe Lymphsystem hingegen befindet sich tief zur Faszie, drainiert die Lymphe der subfaszialen Gewebe (Muskeln, Knochen, Gelenke etc.) und begleitet die tiefen Arterien und Venen. Im weiteren Verlauf wird hier primär auf das oberflächliche Lymphsystem eingegangen.

\section{》) Das Lymphgefäßsystem glie- dert sich in ein oberflächliches und ein tiefes System}

Das oberflächliche Lymphgefäßsystem gliedert sich in sog. Lymphareale, -zonen und -territorien [2]. Bei einem Lymphareal handelt es sich um ein kreisförmiges Hautareal mit einem Durchmesser von maximal $4 \mathrm{~cm}$ (• Abb. 2). Ein Lymphareal beinhaltet ein oberflächliches (kutanes) und ein tiefes (subkutanes) Netz von Lymphkapillaren. Die beiden Kapillarnetze sind durch vertikal verlaufende Lymphkapillaren miteinander verbunden. Lymphareale werden durch sog. Präkollektoren, die im Gegensatz zu den Lymphkapillaren bereits Klappen aufweisen, drainiert. Eine Lymphzone ist ein streifenförmiges Hautgebiet, das aus mehreren Lympharealen besteht und durch einen sog. Kollektor drainiert wird, sodass ein Kollektor die Lymphe von mehreren Präkollektoren aufnimmt (- Abb. 3). Mehrere Lymphzonen vereinigen sich schließlich zu einem Lymphterritorium, also einem größeren Hautareal, das von einem Lymphgefäßbündel drainiert wird. Zwischen 2 benachbarten Territorien besteht keine Verbindung der Lymphgefäße, sodass Territoriengrenzen als "lymphatische Wasserscheiden“ bezeichnet werden können. Einzig über das kutane und subkutane Kapillarnetz der Lymphareale bestehen Verbindungen der Lymphgefäße zwischen benachbarten Territorien.

\section{Kopf und Gesicht}

Im Gesicht selbst sind kaum Lymphknoten zu finden. Die meisten Lymphknoten des Kopfesliegen an der Kopf-Hals-Grenze und bilden hier den Circulus lymphaticus pericervicalis (• Abb. 4). Zu diesem zählen die Nll. occipitales (im Bereich der A. occipitalis und des N. occipitalis major), die Nll. sternocleidomastoidei (unter dem M. sternocleidomastoideus), die Nll. retroauriculares (auf dem Proc. mastoideus), die Nll. parotidei superficiales (am oberen, hinteren und unteren Rand der Parotis), die Nll. parotidei profundi (im präaurikulären Bereich des oberflächlichen Parotislappens), die Nll. submandibulares (im Trigonum submandi-

\begin{tabular}{|ll}
\hline \multicolumn{2}{|l}{ Abkürzungen } \\
\hline A. & Arteria \\
\hline N. & Nervus \\
\hline NI. & Nodus lymphaticus \\
\hline NII. & Nodi lymphatici \\
\hline Proc. & Processus \\
\hline V. & Vena \\
\hline
\end{tabular}




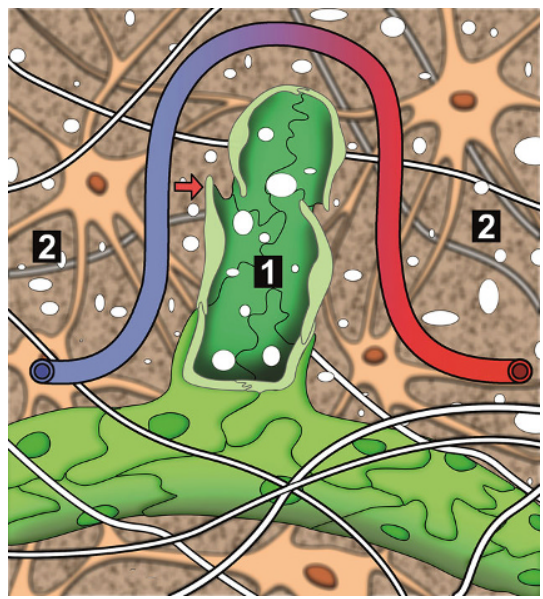

Abb. 1 A Initiale Lymphgefäße: 1 Lymphkapillare (Lymphsinus), 2 prälymphatisches Kanalsystem des Interstitiums, roter Pfeil: interzelluläre Lücke (,schwingender Zipfel“). (Mod. nach [1]; Illustration: A. Bauer. @ Gottfried Schatz Research Center|Makroskopische und klinische Anatomie)

bulare) und die Nll. submentales (in der Regio submentalis). Hierbei sind v. a. die submandibulären Lymphknoten von besonderer Bedeutung, da sie die Lymphe aus dem Großteil der Haut des Gesichts, der Nase, des Kiefers, des Mundbodens, z. T. aus den Nasennebenhöhlen und den Zähnen filtern [1, 5]. Von hier fließt die Lymphe zu den Halslymphknoten ab, die in 2 Gruppen unterteilt werden, die Nll. cervicales anteriores und laterales, wobei die laterale Knotengruppe für den Lymphabfluss aus dem Gesicht bedeutsam ist. Die nächste Lymphknotenstation bilden schließlich die Nll. supraclaviculares, wobei deren efferente Lymphgefäße in den Ductus thoracicus (links) bzw. in den Ductus lymphaticus dexter (rechts) (manchmal auch direkt in den linken bzw. rechten Venenwinkel) einmünden.

\section{》) Die meisten Lymphknoten des Kopfes liegen an der Kopf- Hals-Grenze}

Lymphterritorien existieren zwar auch im Kopf- und Gesichtsbereich (• Abb. 4), weisen jedoch nicht die am restlichen Körper vorhandenen, deutlichen „Wasserscheiden“ auf, da die Kollektoren v. a.

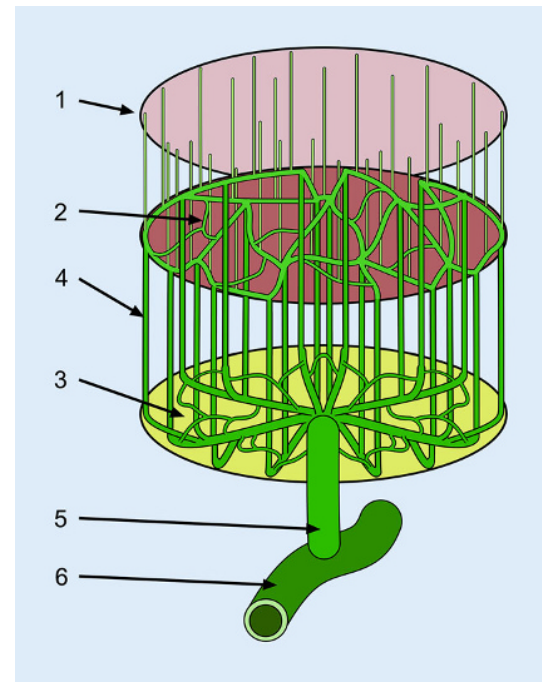

Abb. $2 \Delta$ Lymphareal: 1 Epidermis, 2 oberflächliches (kutanes) Kapillarnetz, 3 tiefes (subkutanes) Kapillarnetz, 4 verbindende Lymphkapillaren, 5 Präkollektor, 6 Kollektor. (Mod. nach [5]; Illustration: A. Bauer. @ Gottfried Schatz Research Center|Makroskopische und klinische Anatomie)

im Gesichtsbereich immer wieder die Seite kreuzen und sich mit den kontralateralen Kollektoren verbinden. Einzig an der Kopfhaut handelt es sich um „echte" Lymphterritorien, wobei ein frontales Territorium (dem Versorgungsgebiet des vorderen Astes der A. temporalis superficialis entsprechend), ein parietales Territorium (dem Versorgungsgebiet des hinteren Astes der A. temporalis superficialis entsprechend) und ein okzipitales Territorium (dem Versorgungsgebiet der A. occipitalis entsprechend) unterschieden werden. Die Lymphe aus dem frontalen Territorium wird in die Nll. parotidei superficiales drainiert, die Gefäßbündel des parietalen Territoriums enden an den retroaurikulären Lymphknoten sowie an den Nll. jugulares interni, während die Lymphe aus dem okzipitalen Territorium in die Nll. occipitales und Nll. sternocleidomastoidei abfließt. Im Gesicht selbst erfolgt die Lymphdrainage in der Weise, dass die Lymphe der Nasenwurzel in die Nll. parotidei superficiales drainiert wird, während die Lymphe des Nasenrückens, der Nasenflügel und der Nasenspitze in die Nll. submandibulares abfließt. Im Bereich der Wange erfolgt eine Unterteilung durch eine Linie- von der Glabella zum Angulus mandibulae verlaufend, also etwa dem Verlauf der A.

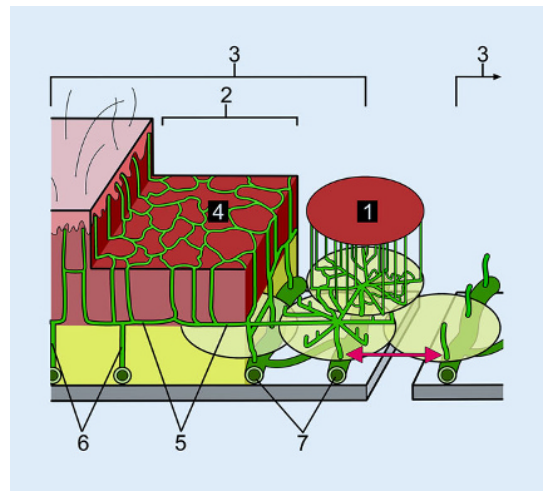

Abb. 3 ॥ 1 Lymphareal, 2 Lymphzone, 3 Lymphterritorium, 4 oberflächliches (kutanes) Kapillarnetz, 5 tiefes (subkutanes) Kapillarnetz, 6 Präkollektoren, 7 Kollektoren, roter Doppelpfeil:Verbindung der Lymphgefäße zwischen 2 Territorien auf Ebene der Kapillarnetze. (Mod. nach [5]; IIlustration: A. Bauer. @ Gottfried Schatz Research Center|Makroskopische und klinische Anatomie)

und V. facialis folgend, in ein vorderes und ein hinteres Lymphgefäßgebiet. Die Lymphe aus dem hinteren Gebiet wird in die Nll. parotidei superficiales drainiert. Das vordere Lymphgefäßgebiet der Wange wird weiter unterteilt in ein infraorbitales Hautgebiet, dessen Lymphe zu den Nll. submandibulares transportiert wird, ein unteres mediales Hautgebiet, dessen Lymphe in die Nll. submentales drainiert wird, und ein unteres laterales Hautgebiet, dessen Lymphe zu den Nll. parotidei superficiales drainiert wird. Die Lymphgefäße der Oberlippe folgen den Ästen der A. und V. facialis und enden an den Nll. submandibulares. Im Bereich der Unterlippe und des Kinns finden sich ein mittleres und 2 seitliche Drainagegebiete, wobei die Kollektoren des mittleren Drittels an den Nll. submentales und die Kollektoren der beiden seitlichen Drittel an den Nll. submandibulares enden.

\section{Hals}

Auch im Bereich des Halses sind keine „echten“ Territorien zu finden $[1,5]$. Aus der Haut der seitlichen Halsregion erfolgt der Lymphabfluss einerseits in die sog. „Akzessoriuskette“, die den N. accessorius im seitlichen Halsdreieck begleitet. 
Zusätzlich erfolgt der Lymphabfluss in die Jugularis-interna-Kette, die entlang der V. jugularis interna verläuft, und in die Nll. supraclaviculares. Die 3 letztgenannten Knotengruppen gehören zu den Nll. cervicales laterales profundi. Aus der vorderen Halsregion erfolgt der Lymphabfluss im oberen (suprahyoidalen) Bereich in die Nll. submentales und weiter nach oben in die Nll. parotidei superficiales, nicht jedoch in die Nll. submandibulares. Aus dem unteren (infrahyoidalen) Bereich der vorderen Halsregion fließt die Lymphe der Haut zu den Nll. jugulares interni und anteriores $\mathrm{ab}$.

\section{Rumpf}

Im Bereich des Rumpfes sind - im Gegensatz zu Gesicht und Hals - die lymphatischen Hautterritorien mit den „Wasserscheiden“" sehr deutlich ausgeprägt [1-3]. So findet man am Rücken - zählt man die Nackenterritorien dazu - insgesamt 6 lymphatische Hautterritorien: je 2 im Nackenbereich, 2 im thorakalen Bereich (obere Rumpfterritorien) und 2 im lumbosakralen Bereich (untere Rumpfterritorien), wobei die Territorien der rechten und linken Seite durch eine vertikal verlaufende „Wasserscheide" voneinander getrennt sind (• Abb. 5). Die horizontal verlaufende „Wasserscheide“ zwischen dem Nackenterritorium und dem oberen Rumpfterritorium verläuft von der Vertebra prominens zum Akromion. Die „Wasserscheide" zwischen dem oberen und unteren Rumpfterritorium verläuft vom Dornfortsatz des 2. Lendenwirbels entlang der Crista iliaca nach vorne. Die Lymphe aus den Nackenterritorien wird in die tiefen, seitlichen Halslymphknoten (Akzessoriuskette) drainiert, die der thorakalen Territorien (oberen Rumpfterritorien) in die axillären Lymphknoten, und die Lymphe der lumbosakralen Territorien (unteren Rumpfterritorien) fließt in die inguinalen Lymphknoten ab. An der Vorderseite des Rumpfes werden pro Seite 2 weitere Territorien unterschieden, die - gleich wie am Rücken - als obere und untere Rumpfterritorien bezeichnet werden (• Abb. 5). Diese sind durch eine lymphatische Wasserscheide, die von der Crista ilia-

J Ästhet Chir 2019 - 12:51-58 https://doi.org/10.1007/s12631-018-0168-x

(c) Der/die Autor(en) 2018

\section{U. Pilsl·F. Anderhuber}

\section{Anatomie des Lymphsystems}

\section{Zusammenfassung}

Das Lymphgefäßsystem des menschlichen Körpers gliedert sich in ein oberflächliches und ein tiefes System. Beide Systeme stehen durch perforierende Lymphgefäße, die die Faszie durchsetzen, miteinander in Verbindung. Dieses Prinzip ist besonders an den Extremitäten sehr gut ausgebildet. Im Bereich des oberflächlichen Lymphsystems finden sich Untereinheiten, die als Lymphareale, Lymphzonen und Lymphterritorien bezeichnet werden. Lymphterritorien sind besonders hervorzuheben, da die Lymphgefäße zwischen 2 benachbarten Territorien keine Verbindung aufweisen, sodass Territoriengrenzen als "Iymphatische
Wasserscheiden" bezeichnet werden. Dies gilt für den Rumpf- und Extremitätenbereich, nicht jedoch für das Gesicht und den Hals, an denen sich Lymphgefäße zweier benachbarter Territorien sehr wohl miteinander verbinden und auch auf die Gegenseite wechseln. Der zentrale Lymphabfluss erfolgt zum Großteil über den Ductus thoracicus, zu einem kleinen Teil über den Ductus lymphaticus dexter in das Venensystem.

Schlüsselwörter

Lymphgefäße · Lymphknoten · Lymphterritorien · Lymphabfluss · Lymphatische Wasserscheide

\section{Anatomy of the lymph system}

\section{Abstract}

The lymphatic system of the human body is subdivided into a superficial and a deep system. Both systems are connected with each other by perforating lymph vessels, which penetrate the fascia. This concept is especially well-developed in the limbs. Within the superficial lymph system subunits known as the lymphatic areas, lymphatic zones and lymphatic territories are found. Lymphatic territories are particularly highlighted, because the lymph vessels between adjacent territories have no connections with each other, so that the territory borders are known as lymphatic watersheds. This is valid for the trunk and the limbs but not for the face and the neck, where the lymph vessels of adjacent territories are indeed connected with each other and also transfer to the opposite side. The central drainage of the lymph into the venous system is carried out mainly by the thoracic duct but also to a minor extent by the right lymphatic duct.

Keywords

Lymphatic vessels · Lymph nodes - Lymphatic territories · Lymph drainage · Lymphatic watershed ca schräg abwärts zum Nabel verläuft, voneinander getrennt. Die Lymphe der vorderen oberen (supraumbilikalen) Rumpfterritorien endet an den Nll. pectorales (einer Untergruppe der axillären Lymphknoten), während die Lymphe der vorderen unteren (infraumbilikalen) Rumpfterritorien zu den oberflächlichen inguinalen Lymphknoten absteigt. Die vorderen und hinteren Rumpfterritorien wiederum sind durch eine lymphatische Wasserscheide, die entlang der mittleren Axillarlinie verläuft, voneinander getrennt.

\section{Extremitäten}

Speziell im Bereich der Extremitäten ist das Prinzip des oberflächlichen und tiefen Lymphgefäßsystems gut erkennbar. Die oberflächlichen Lymphgefäße verlaufen epifaszial, während die tiefen Lymphgefäße subfaszial mit den tiefen Arterien und Venen verlaufen. Das oberflächliche und das tiefe Lymphsystem sind jedoch durch sog. Perforansgefäße, die die Faszie durchbrechen, miteinander in Verbindung (• Abb. 6).

An der oberen Extremität enden sowohl das oberflächliche wie auch das tiefe Lymphsystem an den axillären Lymphknoten, deren Anzahl zwischen 10 und 50 variiert. Nach ihrer topografischen La- 


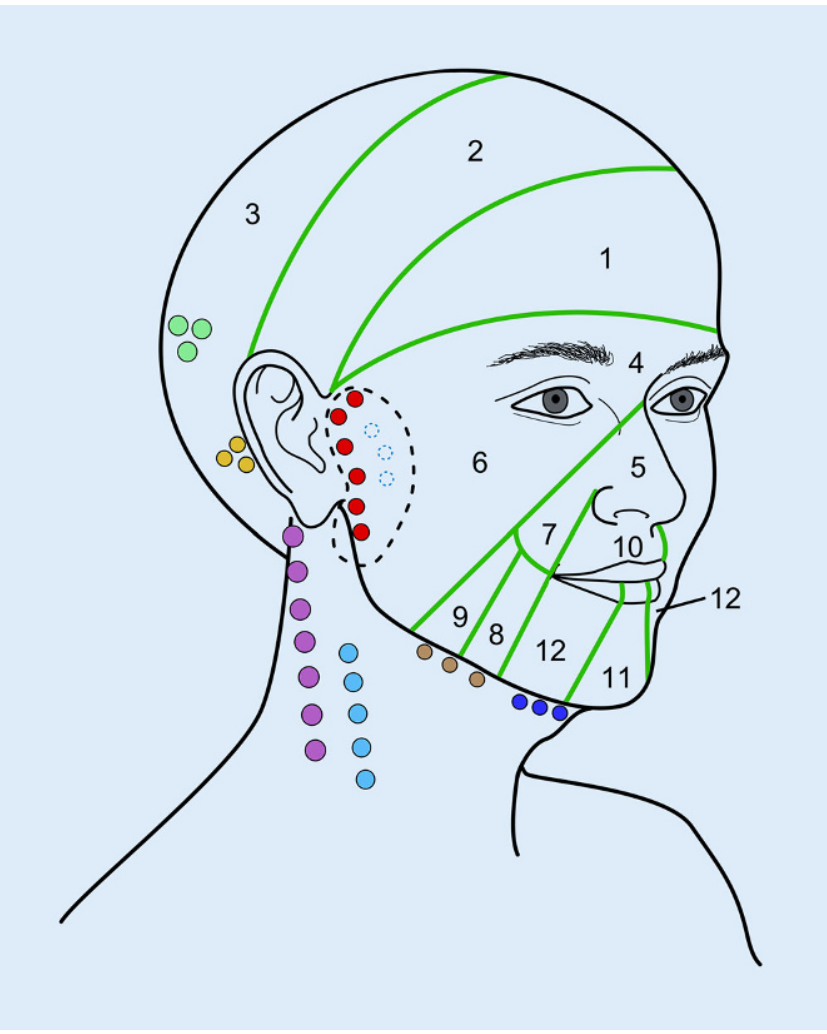

Abb. 4 ム Lymphknoten und Lymphterritorien im Kopf-und Gesichtsbereich. Lymphknoten des Circulus lymphaticus pericervicalis: grün: NII. occipitales, violett: NII. sternocleidomastoidei, gelb: NII. retroauriculares, rot: NII. parotidei superficiales, hellblau-gestrichelt: NII. parotidei profundi, braun: NII. submandibulares, dunkelblau: NII. submentales, hellblau: NII. jugulares interni. Lymphterritorien: 1 frontales Territorium, 2 parietales Territorium, 3 okzipitales Territorium, 4 „Territorium" der Nasenwurzel, 5 „Territorium" des Nasenrückens, der Nasenflügel und der Nasenspitze, 6 hinteres „Territorium “ der Wange, 7 infraorbitales "Territorium" der Wange, 8 unteres mediales "Territorium" der Wange, 9 unteres laterales „Territorium" der Wange, 10 „Territorium" der Oberlippe, 11 mittleres „Territorium" von Unterlippe und Kinn, 12 seitliche „Territorien“ von Unterlippe und Kinn. (Illustration: A. Bauer. @ Gottfried Schatz Research Center|Makroskopische und klinische Anatomie)

ge werden sie in 5 Untergruppen gegliedert. Diese sammeln nicht nur die Lymphe der oberen Extremität, sondern auch der Schulter und der vorderen und hinteren Rumpfwand (oberhalb des Nabels). Betrachtet man das oberflächliche Lymphsystem der oberen Extremität, so erfolgt die Drainage der Lymphe der Handfläche und Finger großteils in die Kollektoren des Handrückens. Von hier wird die Lymphe in zunächst 2 Lymphgefäßbündel an der Dorsalseite des Unterarms, ein ulnares und ein radiales Bündel, drainiert (• Abb. 7). Diese beiden Bündel schlingen sich um den lateralen bzw. medialen Rand des Unterarms auf die Ventralseite. Hier findet sich ein drittes Lymphgefäßbündel, das mediane Bündel. Dieses ist das kleinste der 3 Unterarmbündel und sammelt einen geringen Teil der Lymphe der Handfläche. Alle 3 Unterarmbündel bogens. Am Oberarm findet man erneut 3 Lymphgefäßbündel:

- ein medianes Oberarmbündel, das aus der Vereinigung der 3 Unterarmbündel hervorgeht, somit die gesamte Lymphe der Hand und des Unterarmes aufnimmt und an den axillären Lymphknoten endet;

- das dorsolaterale Oberarmbündel, das die Lymphe aus dem dorsalen und lateralen Bereich der Schulter und des Oberarmes sammelt und sowohl an den infraklavikulären (Untergruppe der axillären Lymphknoten) als auch an den supraklavikulären Lymphknoten endet;

- das dorsomediale Oberarmbündel nimmt die Lymphe aus dem dorsalen und medialen Bereich der Schulter vereinigen sich etwa auf Höhe des Ell-

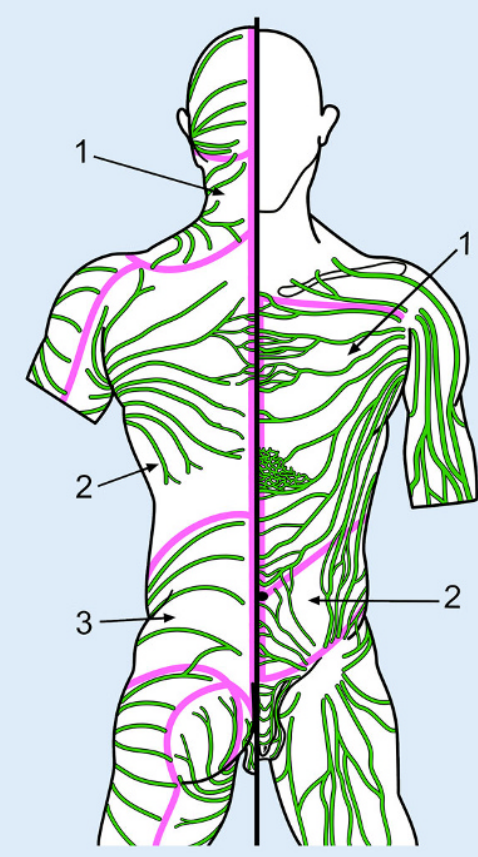

Abb. 5 - Links: Ansicht von dorsal: 1 Nackenterritorium, 2 oberes Rumpfterritorium, 3 unteres Rumpfterritorium. Rechts: Ansicht von ventral: 1 oberes Rumpfterritorium, 2 unteres Rumpfterritorium. (Mod. nach [5]; Illustration: A. Bauer. (c) Gottfried Schatz Research Center| Makroskopische und klinische Anatomie)

und des Oberarmes auf und endet an den axillären Lymphknoten.

Dementsprechend finden sich an der oberen Extremität insgesamt 6 Lymphterritorien ( $\bullet \mathbf{A b b}$. 7): ein radiales, ein ulnares und ein medianes Unterarmterritorium sowie ein medianes, ein dorsolaterales und ein dorsomediales Oberarmterritorium. Die tiefen Lymphgefäße der oberen Extremität begleiten die tiefen Arterien und Venen und sind großteils nach ihnen benannt. So wird die Lymphe der Hand in einen oberflächlichen und tiefen Lymphbogen und von hier in die radialen und ulnaren Kollektoren des Unterarmes, die die A. radialis und ulnaris begleiten, drainiert. Die beiden letztgenannten Bündel vereinigen sich etwa auf Höhe des Ellbogens zu einem Tractus lymphaticus, der mit der 


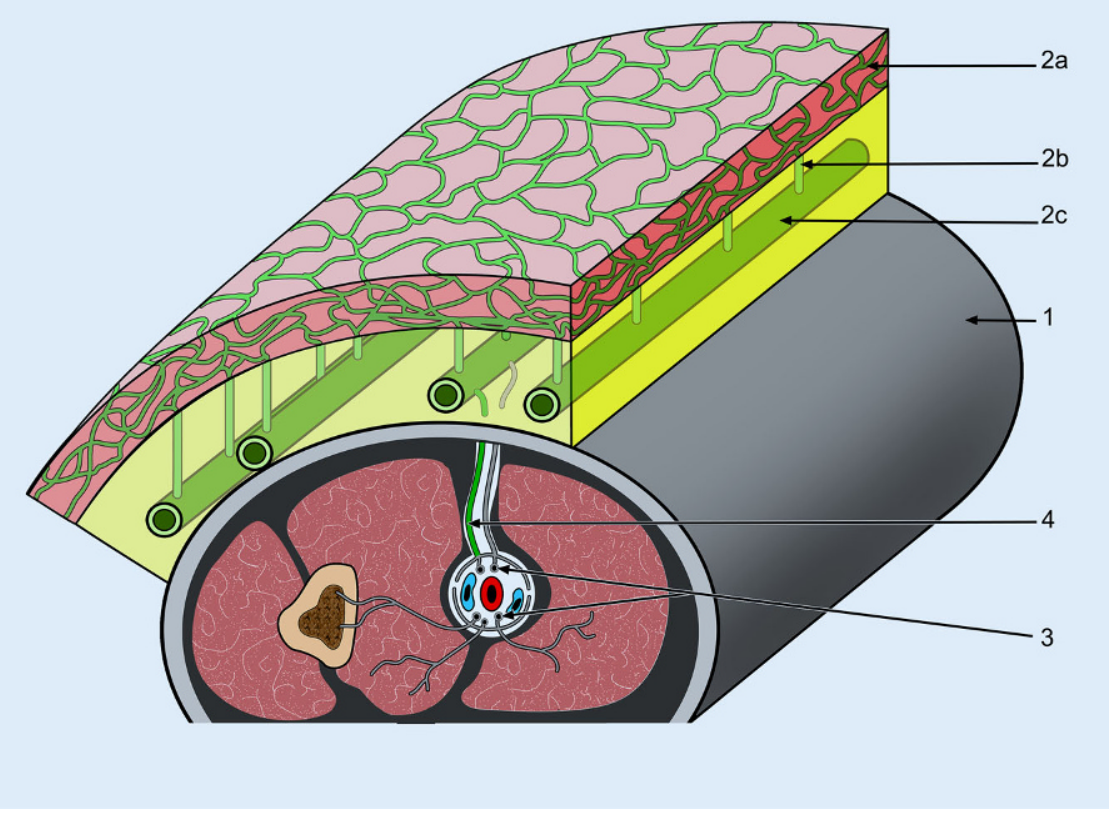

Abb. 6 ॥ Querschnitt durch eine Extremität: 1 Faszie, 2 oberflächliche Lymphgefäße (2a Kapillaren, $2 b$ Präkollektoren, 2cKollektoren), 3 tiefe Lymphgefäße, 4 Perforansgefäße. (Mod. nach [2]; Illustration: A. Bauer. @ Gottfried Schatz Research Center|Makroskopische und klinische Anatomie)

A. brachialis am Oberarm verläuft und an den axillären Lymphknoten endet.

Die Lymphe der unteren Extremität wird in den inguinalen Lymphknoten gesammelt. Hierbei wird zwischen oberflächlichen und tiefen Lymphknoten (Nll. inguinales superficiales et profundi) unterschieden. Die Nll. inguinales superficiales gliedern sich in einen Tractus horizontalis, der parallel zum Leistenband liegt, und einen Tractus verticalis, der sich um die Einmündungsstelle der $\mathrm{V}$. saphena magna in die V. femoralis gruppiert. Diese oberflächlichen Lymphknoten sammeln die Lymphe der unteren Körperhälfte (vom Nabel abwärts), aber auch von den äußeren Genitalien und vom Perineum. Die Nll. inguinales profundi formen Lymphknotenketten entlang der A. und $\mathrm{V}$. femoralis und gehen oberhalb des Leistenbandes in die Nll. iliaci externi über. Der am weitesten proximal gelegene und konstanteste Lymphknoten der tiefen inguinalen Knoten ist der sog. Rosenmüller-Lymphknoten, der in der Lacuna lymphatica gelegen ist. Die tiefen inguinalen Lymphknoten sammeln die Lymphe aus den Knochen, Gelenken und Faszien der unteren Extremität so- wie aus den oberflächlichen inguinalen Lymphknoten.

\section{》) Die Lymphe der unteren Extremität wird in den inguinalen Lymphknoten gesammelt}

Die oberflächlichen Lymphgefäße der unteren Extremität verhalten sich zunächst ähnlich wie an der oberen Extremität, sodass die Lymphe der Fußsohle und der Zehen in die Kollektoren des Fußrückens drainiert wird. Von hier erfolgt der weitere Lymphabfluss in 2 Lymphgefäßbündel. Das ventromediale Lymphgefäßbündel (• Abb. 8) ist das größte Bündel der unteren Extremität. Es folgt dem Verlauf der V. saphena magna, sammelt die Lymphe des Großteils des Fußrückens, des medialen Fußrandes, des vorderen, medialen und z.T. hinteren Anteils des Unterschenkels und des vorderen Anteils des Oberschenkels und endet schließlich an den oberflächlichen inguinalen Lymphknoten. Verglichen mit der Anzahl der Lymphgefäße im Bereich des Unter- und Oberschenkels ist die Anzahl der Gefäße auf Höhe des Kniegelenks relativ gering, weshalb man hier

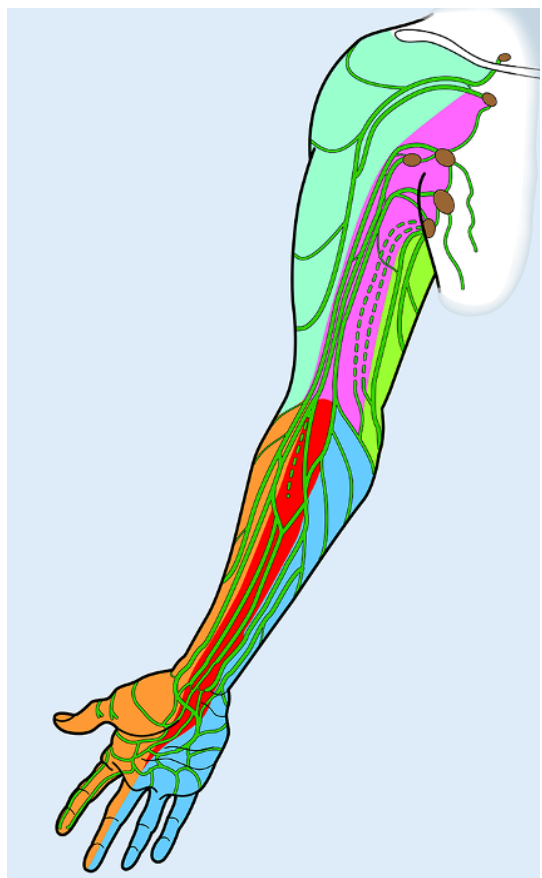

Abb. 7 ॥ Lymphgefäßbündel und Territorien der oberen Extremität. Orange: radiales Unterarmterritorium, blau: ulnares Unterarmterritorium, rot: medianes Unterarmterritorium, pink: medianes Oberarmterritorium, hellgrün: dorsolaterales Oberarmterritorium, dunkelgrün: dorsomediales Oberarmterritorium. (Illustration: A. Bauer. $\odot$ Gottfried Schatz Research Center| Makroskopische und klinische Anatomie)

von einem „physiologischen Flaschenhals" spricht. Ein weiterer Lymphabfluss vom Fuß erfolgt über das wesentlich kleinere dorsolaterale Bündel. Dieses folgt dem Verlauf der V. saphena parva, sammelt die Lymphe zu einem kleinen Teil vom Fußrücken, vom lateralen Fußrand, der Ferse und einem schmalen Streifen im Bereich der Wade und endet bereits in der Kniekehle an den Nll. poplitei superficiales. Zwei weitere Gefäßbündel befinden sich im Oberschenkelbereich: ein dorsolaterales Bündel, das die Lymphe aus den dorsalen und lateralen Regionen des Oberschenkels und der Hüfte sammelt, nach vorne umbiegt und an den Nll. inguinales superficiales endet, und ein dorsomediales Bündel, das die Lymphe aus den dorsalen und medialen Bereichen des Oberschenkels, der Hüfte und der Perinealregion drainiert und - gleich wie das dorsolaterale Bündel nach vorne zu den Nll. inguinales superficiales zieht. Entsprechend finden sich somit an der unteren Extremität insge- 


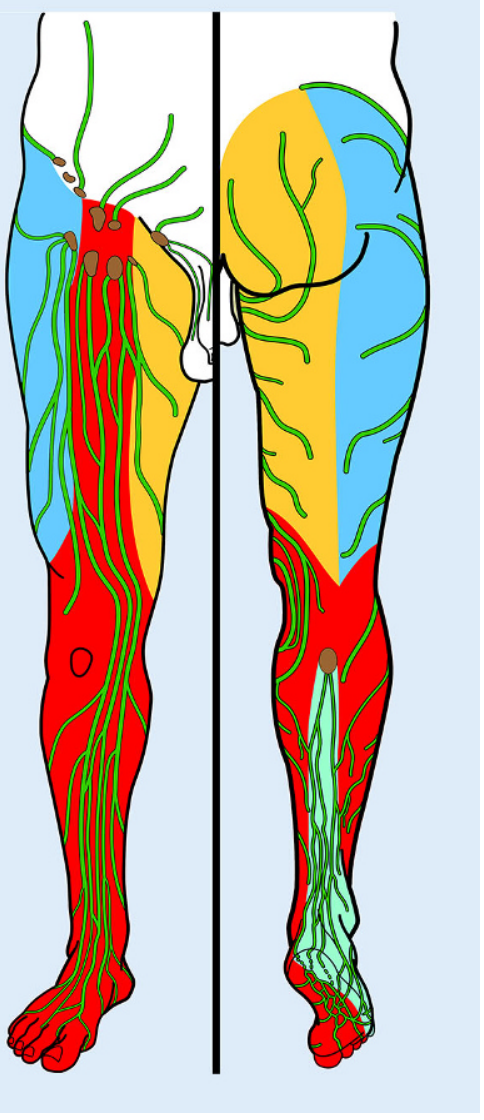

Abb. 8 \& Lymphgefäßbündel und Territorien der unteren Extremität. Links: Ansicht von ventral, rechts: Ansicht von dorsal. Rot:ventromediales Territorium, grün: dorsolaterales Territorium, blau: dorsolaterales Oberschenkelterritorium, gelb: dorsomediales Oberschenkelterritorium (Illustration: A. Bauer. @ Gottfried Schatz Research Center|Makroskopische und klinische Anatomie)

samt 4 Lymphterritorien (• Abb. 8), die wiederum durch „Wasserscheiden“ voneinander getrennt sind: das große ventromediale Territorium und das kleinere dorsolaterale Territorium und im proximalen Bereich das dorsolaterale und das dorsomediale Oberschenkelterritorium mit den oben beschriebenen Drainagegebieten.

Bei den tiefen Lymphgefäßen der unteren Extremität unterscheidet man zwischen Haupt- und Nebenkollektoren. Die Hauptkollektoren begleiten die großen Arterien und Venen, während die $\mathrm{Ne}$ benkollektoren kleineren Arterien, die Muskeln und Gelenke versorgen, folgen. Somit zählen zu den Hauptkollektoren am Unterschenkel die Vasa lymphatica tibialia anteriora und posteriora sowie die

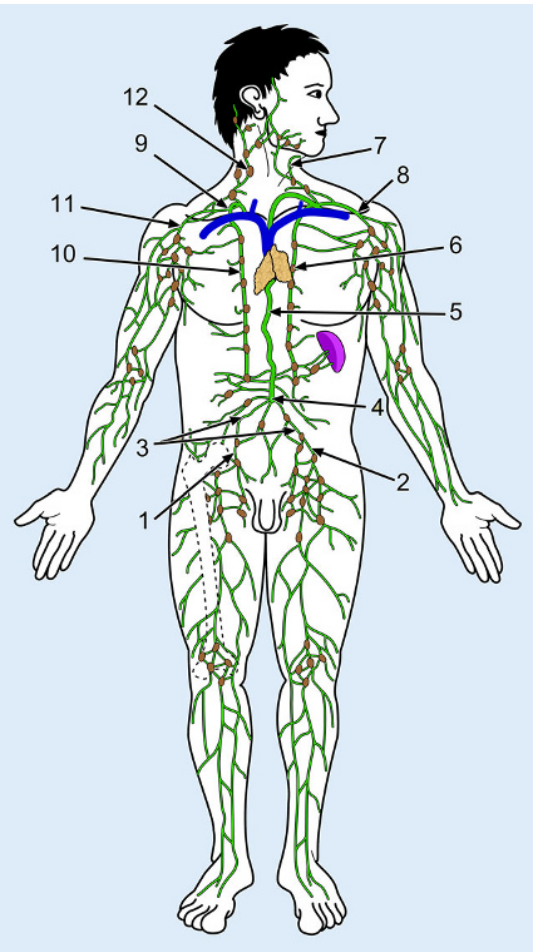

Abb. $9 \Delta$ Zentraler Lymphabfluss: 1 Truncus lumbalis dexter, 2 Truncus lumbalis sinister, 3 Trunci intestinales, 4 Cisterna chyli, 5 Ductus thoracicus, 6 Truncus bronchomediastinalis sinister, 7 Truncus jugularis sinister, 8 Truncus subclavius sinister, 9 Ductus lymphaticus dexter, 10 Truncus bronchomediastinalis dexter, 11 Truncus subclavius dexter, 12 Truncus jugularis dexter. (Illustration: A. Bauer. @ Gottfried Schatz Research Center | Makroskopische und klinische Anatomie)

Vasa lymphatica fibularia und am Oberschenkel die Vasa lymphatica femoralia. Nebenkollektoren sind beispielsweise die Vasa lymphatica femoralia profunda, die die A. profunda femoris begleiten.

\section{Zentraler Lymphabfluss}

Die Lymphe der gesamten unteren Extremität wird im rechten bzw. linken Truncus lumbalis gesammelt (• Abb. 9). Diese beiden Lymphstämme nehmen zusätzlich die Lymphe von den Beckenorganen auf und vereinigen sich mit den Trunci intestinales aus dem Darm zur Cisterna chyli. Diese ist im Bereich des Hiatus aorticus des Zwerchfells hinter der Aorta abdominalis lokalisiert und stellt den Beginn des größten Lymphgefäßes des Körpers, des Ductus thoracicus, dar. Dieser verläuft im hinteren Mediastinum aufwärts und nimmt zunächst den Truncus bronchomediastinalis sinister (mit der Lymphe aus dem linken Brustraum) auf. Anschließend münden in den Ductus thoracicus der Truncus jugularis sinister (mit der Lymphe aus der linken KopfHals-Region) und der Truncus subclavius sinister (mit der Lymphe aus der linken oberen Extremität) ein. Schließlich mündet der Ductus thoracicus in den linken Venenwinkel, der von der V. jugularis interna sinistra und der V. subclavia sinistra gebildet wird, ein. Der Ductus thoracicus transportiert somit drei Viertel der gesamten Körperlymphe! Sein Pendant auf der rechten Seite bildet der Ductus lymphaticus dexter, der den Truncus bronchomediastinalis dexter, den Truncus subclavius dexter und den Truncus jugularis dexter aufnimmt und in den rechten Venenwinkel einmündet. Der Ductus lymphaticus dexter transportiert somit nur ein Viertel der gesamten Körperlymphe.

\section{Fazit für die Praxis}

- Das Lymphgefäßsystem ist bei verschiedenen Erkrankungen primär oder sekundär von pathologischen Veränderungen betroffen. Hierbei seien v. a. das primäre und sekundäre Lymphödem sowie das Lipödem erwähnt.

- Die Kenntnis der Anatomie des Lymphgefäßsystems sowie der entsprechenden Drainagegebiete (Lymphterritorien) der einzelnen Körperregionen ist sowohl für konservative Therapieansätze (z. B. manuelle Lymphdrainage) wie auch für invasive Therapieformen (z. B. lymphschonende Liposuktion) von Bedeutung, um ein zufriedenstellendes Therapieergebnis zu erreichen.

\section{Korrespondenzadresse}

\section{PD Dr. med. univ. Ulrike PilsI}

Gottfried Schatz Zentrum, Makroskopische und klinische Anatomie, Medizinische Universität Graz

Harrachgasse 21/1, 8010 Graz, Österreich ulrike.pils!@medunigraz.at 
Funding. Open access funding provided by Medical University of Graz.

\section{Einhaltung ethischer Richtlinien}

Interessenkonflikt. U. Pilsl gibt an, dass kein Interessenkonflikt besteht.

Dieser Beitrag beinhaltet keine von den Autoren durchgeführten Studien an Menschen oder Tieren.

Open Access Dieser Artikel wird unter der Creative Commons Namensnennung 4.0 International Lizenz (http://creativecommons.org/licenses/by/4.0/deed. de) veröffentlicht, welche die Nutzung, Vervielfältigung, Bearbeitung, Verbreitung und Wiedergabe in jeglichem Medium und Format erlaubt, sofern Sie den/die ursprünglichen Autor(en) und die Quelle ordnungsgemäßnennen, einen Linkzur Creative Commons Lizenz beifügen und angeben, ob Änderungen vorgenommen wurden

\section{Literatur}

1. Földi M, Földi E (2010) Lehrbuch Lymphologie, 7. Aufl. Elsevier, Urban \& Fischer, München

2. Lang J, Wachsmuth W (1982) Praktische Anatomie. Ein Lehr- und Hilfsbuch der Anatomischen Grundlagen ärztlichen Handelns Bd. 7, 7. Teil. Springer, Berlin-Heidelberg-New York

3. Loeweneck H, Feifel G (1993) Praktische Anatomie. Ein Lehr- und Hilfsbuch der Anatomischen Grundlagen ärztlichen Handelns Bd. 2, 6. Teil. Springer, Berlin-Heidelberg-New York

4. Sadler TW (2008) Medizinische Embryologie. Die normale menschliche Entwicklung und ihre Fehlbildungen, 11. Aufl. Thieme, Stuttgart-New York

5. von Lanz T, Wachsmuth W (1955) Praktische Anatomie. Ein Lehr- und Hilfsbuch der Anatomischen Grundlagen ärztlichen Handelns Bd. 1, 2. Teil. Springer, Berlin-Göttingen-Heidelberg

6. Weissleder $\mathrm{H}$, Schuchhardt C (2015) Erkrankungen des Lymphgefäßsystems, 6. Aufl. Vital Verlag, Köln

\section{März 2019}

München 13.-16.03.2019

46. Jahrestagung der Arbeitsgemeinschaft Dermatologische Forschung e.V.

Auskunft: info@adf-online.de, https://www.adf-online.de/

Frankenthal 15.-17.03.2019

DERM 2019

Themen: Berufspolitik, Onkologie, neue Kooperationsformen, Haftpflicht- und Rechtsfragen,

Praxismanagement, OP-Kurs, Fachliche Workshops,

Ästhetik, Laser

Wiss. Leitung: Herr Prof. Dr. med. Klaus Fritz

Auskunft: Frau Angelika Morio, Periderm GmbH,

Postfach 1132, 76866 Kandel,

Fon: 063419350523, Kongress-derma@periderm.com

https://www.dermatologische-praxis.com/home/

Düsseldorf 16.03.2019

14. Jahrestagung der Sektion Handchirurgie der

DGU

Themen: Orthopädie/Unfallchirurgie, Plastische/

Ästhetische Chirurgie; Spezialgebiet: Hand

Wiss. Leitung: Prof. Dr. med. Wolfgang Linhart, Prof.

Dr. med. Joachim Windolf

Auskunft: Herr Marc Dittes, Intercongress $\mathrm{GmbH}$,

Ingeborg-Krummer-Schroth-Str. 30, 79106 Freiburg

Fon: +497616969925,

marc.dittes@intercongress.de,

https://www.intercongress.de

Erlangen 22.03.2019

Septumchirurgie - Grundlagen der Rhinoplastik

- Erlanger Operationskurs

Interaktiver Videoinstruktionskurs

Wiss. Leitung: Prof. Dr. med. Dr. h. c. H. Iro, Prof. Dr. med. J. Wurm

Auskunft: Frau Birgit Lenz, Universitätsklinikum

Erlangen, Waldstr. 1, D-91054 Erlangen,

Fon: 0049 (0) 9131-85-33631,

hno-kurssekretariat@uk-erlangen.de,

www.hno-klinik.uk-erlangen.de

München 26.-29.03.2019

136. Kongress der Deutschen Gesellschaft für

Chirurgie 2019

Themen: Chirurgie, Kinderchirurgie, Mund-

Kiefer-, Gesichtschirurgie, Plastische \& Ästhetische

Chirurgie, Herz- und Thoraxchirurgie, Unfallchirurgie,

Traumatologie, Handchirurgie

Wiss. Leitung: Prof. Dr. M. Anthuber

Auskunft: Frau Franziska Klein, MCN Medizinische

Congressorganisation Nürnberg AG, Neuwieder Str. 9

90411 Nürnberg

Fon: 0911-39316-42

klein@mcn-nuernberg.de

\section{April 2019}

Stuttgart 08.04.2019

8th Annual Meeting of the Rhinoplasty Society of Europe

Wiss. Leitung: N. Cerkes, Istambul; W. Gubisch, S.

Haack, Stuttgart; E. Robotti, Bergamo

Auskunft: boeld communication $\mathrm{GMbH}$, Adlzreiter-

str. 29, 80337 München

Fon: +40 (0)891890 460

www.rhinoplastysociety.eu

Stuttgart 09.-11.04.2019

27th Stuttgart Advanced Course for Functional \&

Aesthetic Rhinoplasty

Wiss. Leitung: Sebastian Haack, Wolfgang Gubisch,

Helmut Fischer

Auskunft: boeld communication GMBH, Adlzreiter-

straße 29, 80337 München,

Fon: +49 (0)8918904616

congress@bb-mc.com,

http://stuttgart-rhinoplasty-2019.de/
Stuttgart 12.-13.04.2019

7th SOS Symposium

International Symposium for Plastic Surgeons |

Salvage procedures after Pitfalls in Aestetic Surgery

Wiss. Leitung: Wolfgang Gubisch \& Sebastian Haack,

Stuttgart - Germany; Christoph Heitmann, Munich -

Germany; Dirk Richter, Cologne - Germany

Auskunft: boeld communication $\mathrm{GmbH}$, Adlzreiter-

straße 29, 80337 München,

Fon: +40 (0)891890460,

http://www.sos2019.eu/

\section{Mai 2019}

Berlin 01.-04.05.2019

50. DDG-Tagung

50. Haupttagung der Deutschen Dermatologischen

Gesellschaft

Wiss. Leitung: Prof. Dr. Dr. L. Bruckner-Tuderman, Prof. Dr. T. Biedermann

Auskunft:e.schmeckenbecher@derma.de,

https://www.derma.de/de/fuer-aerzte/50-ddg-tagung/

München 16.-18.05.2019

XIX. Frühjahrsakademie VDÄPC 2019

Von der Planung zum Eingriff - sichere Ästhetische Chirurgie

Wiss. Leitung: Prof. Dr. Hisham Fansa, Dr. Dominik von Lukowicz

Auskunft: boeld communication $\mathrm{GmbH}$, Adlzreiter-

straße 29, 80337 München,

Fon: +40 (0)89 189046 0, http://www.vdaepc2019.de

Frankfurt/M. 24.-25.05.2019

1. Deutscher Rhinoplastikkongress

Themen: Rhinoplastik-Grundlagen, Septum, mittleres

Gewölbe, knöchernes Nasengerüst, Nasenspitze,

Korrektur des Nasenrückens, Revisions-Chirurgie

Wiss. Leitung: Dr. Nuri Alamuti - Wiesbaden, Dr. Eckart

Buttler - München, Dr. Sebastian Haack - Stuttgart,

Dr. Milos Kovacevic - Hamburg, Prof. Dr. Frank Riedel

- Mannheim, Prof. Dr. Jochen Wurm - Erlangen

Auskunft: Frau Laura Schessl, boeld communication

GmbH, Adlzreiterstraße 29, 80337 München,

Fon: +40 (0)89 1890460 ,

kontakt@rhinoplastik-kongress.de,

https://www.rhinoplastik-kongress.de/

\section{Juni 2019}

Milan 10.-15.06.2019

24th World Congress of Dermatology (WCD)

Auskunft: Organising Secretariat Triumph Italy,

wcd2019milan@thetriumph.com,

http://www.wcd2019milan.org/

Rennes, France 18.-21.06.2019

CARS 2019 Computer Assisted Radiology and

Surgery

33rd International Congress and Exhibition

Themen: Medical Imaging, Computed Maxillofacial

Imaging, Image Processing and Visualization, PACS

and IHE, Telemedicine and E-Health, Computer Aided

Diagnosis, Computer Assisted Radiation Therapy,

Image and $\mathrm{M}$

Wiss. Leitung: Prof. Heinz U. Lemke

Auskunft: Frau Franziska Schweikert CARS Confe-

rence Office, Klettgaustr. 20, 79790 Kuessaberg

Fon: +497742922 434

office@cars-int.org, https://www.cars-int.org

\section{August 2019}

Köln 24.-25.08.2019

8. Sommerakademie der Dermatologischen

Wissenschaft- und Fortbildungsakademie NRW (DWFA)

Auskunft: Rheinisch-Westfälische Dermatologische

Gesellschaft e.V.

https://www.dwfa.de 
Hier steht eine Anzeige.

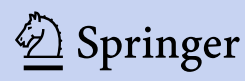

